\title{
Electromagnetic signatures of dark photon superradiance
}

\author{
Andrea Caputo $\odot,{ }^{1,2,3}$ Samuel J. Witte $\odot,{ }^{4}$ Diego Blas, ${ }^{5}$ and Paolo Pani ${ }^{6}$ \\ ${ }^{1}$ School of Physics and Astronomy, Tel-Aviv University, Tel-Aviv 69978, Israel \\ ${ }^{2}$ Department of Particle Physics and Astrophysics, Weizmann Institute of Science, Rehovot 7610001,Israel \\ ${ }^{3}$ Max-Planck-Institut für Physik (Werner-Heisenberg-Institut), \\ Föhringer Ring 6, 80805 München, Germany \\ ${ }^{4}$ Gravitation Astroparticle Physics Amsterdam (GRAPPA), Institute for Theoretical Physics Amsterdam \\ and Delta Institute for Theoretical Physics, University of Amsterdam, \\ Science Park 904, 1098 XH Amsterdam, Netherlands \\ ${ }^{5}$ Theoretical Particle Physics and Cosmology Group, Department of Physics, King's College London, \\ Strand, London WC2R 2LS, United Kingdom \\ ${ }^{6}$ Dipartimento di Fisica, "Sapienza" Università di Roma \& Sezione INFN Romal, \\ Piazzale Aldo Moro 5, 00185 Roma, Italy
}

(Received 1 March 2021; accepted 8 July 2021; published 5 August 2021)

\begin{abstract}
Black hole superradiance is a powerful tool in the search for ultralight bosons. Constraints on the existence of such particles have been derived from the observation of highly spinning black holes, absence of continuous gravitational-wave signals, and of the associated stochastic background. However, these constraints are only strictly speaking valid in the limit where the boson's interactions can be neglected. In this work we investigate the extent to which the superradiant growth of an ultra-light dark photon can be quenched via scattering processes with ambient electrons. For dark photon masses $m_{\gamma^{\prime}} \gtrsim 10^{-17} \mathrm{eV}$, and for reasonable values of the ambient electron number density, we find superradiance can be quenched prior to extracting a significant fraction of the black-hole spin. For sufficiently large $m_{\gamma^{\prime}}$ and small electron number densities, the in-medium suppression of the kinetic mixing can be efficiently removed, and quenching occurs for mixings $\chi_{0} \gtrsim \mathcal{O}\left(10^{-8}\right)$; at low masses, however, in-medium effects strongly inhibit otherwise efficient scattering processes from dissipating energy. Intriguingly, this quenching leads to a time- and energy-oscillating electromagnetic signature, with luminosities potentially extending up to $\sim 10^{56} \mathrm{erg} / \mathrm{s}$, suggesting that such events should be detectable with existing telescopes. As a by-product we also show that superradiance cannot be used to constrain a small mass for the Standard Model photon.
\end{abstract}

DOI: 10.1103/PhysRevD.104.043006

\section{INTRODUCTION}

Black hole $(\mathrm{BH})$ superradiance is the process by which low-energy bosons can extract the rotational energy of a spinning BH [1-6] (see Ref. [7] for an overview). This process is at play for modes with frequency $\omega<m \Omega$, where $\Omega$ is the angular velocity of the $\mathrm{BH}$ and $m$ the azimuthal quantum number of the mode. If this radiation is confined near the $\mathrm{BH}$, the energy extraction may happen at an exponential rate, leading to a so-called "BH bomb" instability [4]. It has long been appreciated that the very mass of a particle can serve as such a confining mechanism [7-10], as Kerr BHs have quasibound state orbits with

Published by the American Physical Society under the terms of the Creative Commons Attribution 4.0 International license. Further distribution of this work must maintain attribution to the author(s) and the published article's title, journal citation, and DOI. Funded by SCOAP ${ }^{3}$. support within and just outside the ergoregion for particles with Compton wavelength roughly comparable to the $\mathrm{BH}$ horizon.

This phenomenon has garnered much attention over the last decade as the search for exotic light particles, such as axions and dark photons, has intensified. Most studies thus far have focused on understanding the growth and evolution of a BH-boson condensate forming through the superradiant instability, under the simplifying assumption that the boson field is noninteracting. In this case, and if the boson mass $m_{b} \lesssim M^{-1}$ (being $M$ the mass of the $\mathrm{BH}$; we use $G=c=\hbar=\kappa_{B}=1$ units hereafter), one expects the superradiant boson cloud to be able to extract up to $\approx 10 \%$ of the angular momentum of a highly spinning $\mathrm{BH}$ over extremely short timescales [11-13]. The presence of gaps in the BH mass-spin "Regge" plane could then serve as an indirect observation of the existence of such bosons $[14,15]$; equivalently, the observation of highly spinning $\mathrm{BHs}$ can be used to constrain the existence of exotic 
particles [16-23]. In addition, if the boson cloud remains bound to the $\mathrm{BH}$ after the superradiant condition has saturated (i.e., when the $\mathrm{BH}$ spin has been reduced to a point where $\omega \simeq m \Omega$ ), its (spinning) dipolar structure produces nearly continuous quadrupolar gravitational waves at a frequency set by the boson mass. Negative searches for such continuous signals [16,17] and for the stochastic background produced by unresolved sources [1821] in LIGO and Virgo have set some further constraints on the mass of ultra-light bosons in a narrow range around $m_{b} \sim$ $10^{-13} \mathrm{eV}$ [22]. Future space-based interferometers such as LISA will probe much smaller masses $[18,19]$ and could also detect other effects, such as the gravitational and environment effects of the bosonic cloud on the evolution of extreme mass-ratio inspirals [24-27]. Overall, current and future (both electromagnetic and gravitational-wave) probes of superradiance can explore the ultralight boson frontier roughly in the range $m_{b} \in\left(10^{-21}, 10^{-11}\right) \mathrm{eV}$, see [7] for a summary of the latest constraints and for their dependence on the particle's spin.

The above description of the superradiant instability is strictly speaking valid only when the boson field is noninteracting. However, superradiance generates enormous number densities, and thus interactions that would conventionally be considered as weak may be sufficient to destroy or quench the evolution of the superradiant cloud. The investigation of quenching mechanisms, and complementary signatures that may arise even when the $\mathrm{BH}$ spin is not significantly altered, has received attention in recent years $[14,15,28-33]$. Given the stringent nature of the superradiance constraints, and the enormous energy densities typically involved in the problem, understanding when and how various models undergo quenching is of utmost importance.

We focus here on the role of ultralight dark photon interactions during the superradiant growth, identifying parameters and model-dependent features for which quenching occurs, and illustrating that electromagnetic signatures may arise when quenching is important. Models featuring the existence of a dark photon are ubiquitous in extensions of the Standard Model (SM) of particle physics [34-37]. Frequently referred to as the "vector portal," these models present a very generic possibility-together with the Higgs and the neutrino portals - to broadly characterize connections between the SM and dark sectors [38]. Over the last several years, the vector portal has become one of the most important paradigms in particle physics models, and is routinely invoked to explain existing experimental anomalies in high-energy physics and cosmology, see, e.g., [39-47]. It is then relevant not to leave any stone unturned, and explore the diverse phenomenological consequences arising from BH superradiance in such models.

We find that despite an initial in-medium suppression $[48,49]$ of the interaction strength (especially relevant at low dark-photon masses), dark photons which kinetically mix with the SM photon will quench if their mass $m_{\gamma^{\prime}} \gtrsim$ $10^{-16} \mathrm{eV}$ and vacuum mixing $\chi_{0} \gtrsim 10^{-7}$ (quenching may still occur for smaller mixings at larger masses, and larger mixings at masses down to $\sim 10^{-17} \mathrm{eV}$, however the details depend on the local electron number density which can span many orders of magnitude). Furthermore, we argue that the superradiant growth of such particles may give rise to a unique, time-dependent, electromagnetic signature that may be detectable using current telescopes.

The outline of this paper is as follows. In Sec. II we present an overview of vector superradiance. We then present the dark photon model in Sec. III. In Sec. IV we discuss the relevant scattering processes that are responsible for inhibiting the growth of the cloud, and estimate the luminosity from the semi-Compton scattering off, and synchrotron emission of, the ambient electrons. We then discuss additional model-dependent quenching mechanisms that do not rely on the existence of the kinetic mixing. Section V includes a brief discussion on implications for the SM photon, with particular reference to implications for superradiant bounds that have been derived on the potential existence of a bare photon mass. We conclude in Sec. VI.

\section{VECTOR SUPERRADIANT INSTABILITY}

We consider the dynamics of a light vector boson $A^{\prime}$ with mass $m_{\gamma^{\prime}}$ on the background of a rotating $\mathrm{BH}$ with mass $M$ and dimensionless spin $\tilde{a} \equiv J / M^{2}$, where $J$ is the angular momentum of the BH. Assuming that the vector field is noninteracting and neglecting backreaction on the metric, its evolution is governed by the Proca equation, $\nabla_{\sigma} F^{\prime \sigma \nu}-m_{\gamma^{\prime}}^{2} A^{\prime \nu}=0$, on the Kerr spacetime. Originally, the superradiant instability in this case was studied in the Fourier domain and in the slow-rotation approximation [50] up to $\mathcal{O}\left(\tilde{a}^{2}\right)$ by expanding the field in a basis of vector spherical harmonics with indices $(l, m)$, yielding a system of ODEs in which modes with different $l$ number and opposite parity are coupled to each other [51,52]. More recently, the eigenvalue problem was solved for arbitrary values of $\tilde{a}$ both analytically in the Newtonian approximation $\left(\alpha \equiv m_{\gamma^{\prime}} M \ll 1\right)$ [53,54], and numerically in the generic case, either by solving a system of PDEs [54,55] or by using the recently discovered separability [56] of the Proca equations in the Kerr metric [56,57]. The Newtonian approximation is typically sufficiently accurate to capture the order of magnitude of the instability timescale when $\alpha \lesssim 0.1$. For a mode with $\omega=\omega_{R}+i \omega_{I}$, the real part and imaginary parts of the frequency are approximately given by [51-53]

$$
\omega_{R}^{2} \simeq m_{\gamma^{\prime}}^{2}\left(1-\left(\frac{\alpha}{l+n+S+1}\right)^{2}\right),
$$




$$
\omega_{I}=\frac{2 \gamma_{S l}}{M} r_{+}\left(m \Omega-\omega_{R}\right) \alpha^{4 l+5+2 S},
$$

where $r_{+}=M\left(1+\sqrt{1-\tilde{a}^{2}}\right)$ is the horizon radius, $\Omega=\tilde{a} /\left(2 r_{+}\right), l=1,2, \ldots$ is the total angular momentum number of the mode, $m$ is the azimuthal number (such that $|m| \leq l), n=0,1,2, \ldots$ is the overtone number, $S=$ $-1,0,1$ the polarization, and $\gamma_{S l}$ a numerical coefficient [7]. The fastest growing mode corresponds to $S=-1$, $l=1$ (yielding $\gamma_{-11}=4$ ) and the superradiant timescale is approximately given by $[51,52]$

$$
\tau_{s} \simeq \frac{M \alpha^{-7}}{\tilde{a} \gamma_{-11}} \simeq \frac{10^{2}}{\tilde{a}}\left(\frac{M}{10 M_{\odot}}\right) \mathrm{s},
$$

where in the last step we assumed a fiducial value $\alpha \sim 0.1$. The typical radius of the superradiant cloud is roughly given by $r_{\text {cloud }} \simeq M / \alpha^{2}$, and ranges from $r_{\text {cloud }} \sim 100 M$ for the fiducial value above down to $r_{\text {cloud }} \sim 10 M$ for $\alpha \sim 0.3$.

If efficient, i.e., if $\tau_{s}$ is small relative to the rate at which the BH's angular momentum grows (e.g., through accretion), the superradiant cloud will grow until it extracts sufficient amount of the angular momentum and saturates the superradiant condition; in the case of a nearly extremal $\mathrm{BH}$, this occurs after the cloud has extracted up to $\approx 10 \%$ of the BH total energy [11-13]. Given a cloud with mass $M_{\text {cloud }}$, the angular momentum loss is $\Delta J=m M_{\text {cloud }} / \omega_{R}$ [7]. Thus, assuming such light degrees of freedom exist, one should not observe highly spinning $\mathrm{BHs}$ with $M \sim\left(2 m_{\gamma^{\prime}}\right)^{-1}$; or equivalently, since no such feature has been observed so far, the existence of highly spinning BHs can be used to constrain these light vector bosons. The $\mathrm{BH}$ accretion rate, controlling the characteristic timescale over which one can adopt a constant $\mathrm{BH}$ mass, can be conservatively estimated to be given by a fraction of the Eddington accretion timescale,

$$
\tau_{\mathrm{Edd}}=\frac{M}{\dot{M}} \sim \frac{\epsilon \sigma_{T}}{4 \pi m_{p}} \sim 1.4 \epsilon \times 10^{15} \mathrm{~s},
$$

where $\sigma_{T}$ is the Thomson cross section, $m_{p}$ the proton mass, and $\epsilon$ an efficiency factor. The timescale above with $\epsilon=\mathcal{O}(1)$ can be considered as a conservative lower bound, since accretion can be much less efficient. We notice that the Eddington timescale is much larger than the superradiant timescale of interest, Eq. (3). Therefore we can safely neglect accretion in the rest of the work.

The superradiant growth can be impeded, or quenched, should processes exist that deplete the abundance of the ultralight bosons at a rate faster than that of superradiant growth, or should the mass, and thus the bound states, of the boson be significantly modified. It is important to emphasize that the interaction responsible for the quenching does not need to be considered by conventional standards to be strong - the very large energy densities achieved during the superradiant growth can often compensate for highly suppressed scattering processes.

\section{THE DARK PHOTON IN A PLASMA}

Dark photons interact with the SM through a kinetic mixing with the SM photon [34]. Working in a basis in which the quadratic action is diagonal, the Lagrangian reads

$$
\begin{aligned}
\mathcal{L}= & -\frac{1}{4} F_{\mu \nu} F^{\mu \nu}-\frac{1}{4} F_{\mu \nu}^{\prime} F^{\prime \mu \nu} \\
& +m_{\gamma^{\prime}}^{2} A_{\mu}^{\prime} A^{\prime \mu}-e J^{\mu}\left(A_{\mu}+\sin \chi_{0} A_{\mu}^{\prime}\right),
\end{aligned}
$$

where $F_{\mu \nu}$ and $F_{\mu \nu}^{\prime}$ are the field strength tensors of the photon and dark photon, respectively, $\sin \chi_{0}$ is the bare kinetic mixing (assumed to be always much smaller than unity, $\left.\sin \chi_{0} \ll 1\right), m_{\gamma^{\prime}}$ is the dark photon mass, and $J^{\mu}$ is a $\mathrm{SM}$ electric current. In this basis we therefore have a direct coupling between the hidden photon and the SM electric current. Notice that here we indicate by $A_{\mu}^{\prime}$ the dark interaction eigenstate. The propagating dark state, found after the diagonalization of the mass matrix, will be instead named $A_{\mathrm{dp}}$.

For large dark photon masses, one can effectively decouple the Proca field solution from the intrinsic dynamics of the electrons and ions (that is to say, the motion of the electrons may be driven by the presence of the dark photon, but the dark photon is not affected by the presence of the plasma); in the small mass limit, however, the motion of these particles can induce in-medium effects $[48,49]$ which cause, e.g., the dark photon and photon to fully decouple in the $m_{\gamma^{\prime}} \rightarrow 0$ limit [58]. Thus, in this case it is necessary to jointly solve for the dynamics of the entire system. ${ }^{1}$

The motion of the electrons (and ions, which we neglect in what follows due to the fact that their velocity is much lower than that of the electrons) in the presence of an electromagnetic field, including the effect of electron-ion collisions and assuming a cold plasma (i.e., with temperature $T \ll m_{e}$ ), is given by

$$
\begin{aligned}
& \frac{\partial \vec{p}_{e}}{\partial t}+\left(\vec{v}_{e} \cdot \nabla\right) \vec{p}_{e} \\
& \quad=-e\left(\vec{E}+\sin \chi_{0} \vec{E}^{\prime}+\vec{v}_{e} \times\left(\vec{B}+\sin \chi_{0} \vec{B}^{\prime}\right)\right)-\nu\left(\vec{p}_{e}-\vec{p}_{i}\right)
\end{aligned}
$$

\footnotetext{
${ }^{1}$ Recently the linearized dynamics of the SM electromagnetic field in a cold plasma and in curved spacetime has been studied in detail [59]. Although the dynamics is quite rich, the final result in terms of quasibound states is in qualitative agreement with what predicted by the dispersion relation of plane waves. In the following we shall therefore use the latter, much simpler approach, postponing a complete dynamical analysis of the system to future work.
} 
where $\vec{v}_{e}$ and $\vec{p}_{e}$ are the velocity and momentum of the electrons, $\vec{p}_{i}$ the momentum of the ions (which in the rest frame can be expressed as $-\vec{p}_{e}$ ), and $\nu$ is the electron-ion collision frequency. This equation must be solved simultaneously with the electromagnetic and Proca equations, given by

$$
\begin{gathered}
\square A^{\mu}=e J^{\mu}, \\
\square A^{\prime \mu}+m_{\gamma^{\prime}}^{2} A^{\prime \mu}=e \sin \chi_{0} J^{\mu} .
\end{gathered}
$$

In full generality this problem is intractable, and thus in what follows we identify various limiting regimes of interest and use these solutions to determine how the dark photon field will evolve.

\section{A. Collisional regime}

Let us first consider the effect of electron-ion collisions (note that the propagation of the ultralight dark photon in this regime was first studied by [49]), the frequency of which is given by

$$
\nu \simeq \frac{n_{e} e^{4} \log \Lambda}{2 \pi m_{e}^{2} v_{e}^{3}},
$$

where $n_{e}$ and $m_{e}$ are the electron number density and mass, $v_{e}$ is the electron velocity in the rest frame of the proton, and $\Lambda$ is the Coulomb logarithm which takes on a value of $\log \Lambda \sim 10$ in plasmas of interest. The typical order of magnitude of $n_{e}$ near an astrophysical $\mathrm{BH}$ is discussed in Appendix A. To remain agnostic relative to the uncertainties of the ambient density and accretion flow, we shall assume $n_{e} \in\left(10^{-4}, 10^{4}\right) \mathrm{cm}^{-3}$. Correspondingly, the plasma frequency

$$
\omega_{p}=\sqrt{\frac{4 \pi n_{e} e^{2}}{m_{e}}} \approx 10^{-13}\left(\frac{n_{e}}{10^{-4} \mathrm{~cm}^{-3}}\right)^{1 / 2} \mathrm{eV},
$$

ranges from $10^{-9} \mathrm{eV}$ to $10^{-13} \mathrm{eV}$. $^{2}$

The characteristic timescale for electron-ion collisions

$$
\tau_{e i}=\nu^{-1} \sim\left(\frac{10^{4} \mathrm{~cm}^{-3}}{n_{e}}\right)\left(\frac{v_{e}}{10^{-3}}\right)^{3} \mathrm{~s}
$$

should be compared with the oscillation period of the electric field,

$$
\frac{2 \pi}{\omega_{R}} \sim 4 \times 10^{-4}\left(\frac{10^{-11} \mathrm{eV}}{m_{\gamma^{\prime}}}\right) \mathrm{s},
$$

\footnotetext{
${ }^{2}$ As originally recognized in Refs. [60,61], the plasma frequency can be in the mass scale for which superradiance is effective for astrophysical BHs in various mass ranges. We note, however, that in our case superradiance is not plasma-induced, but triggered by the bare mass of the dark photon.
}

to determine whether collisions are important. In the absence of collisions (i.e., when $\tau_{e i} \gg 2 \pi / \omega_{R}$ ), an oscillating electric field will induce oscillations in the plasma; the net work done on the plasma over a period of oscillation however will be zero, and thus energy in the field is not dissipated. As collisions become important (i.e., when $\left.\tau_{e i} \lesssim 2 \pi / \omega_{R}\right)$, electrons and ions accelerated by the fields scatter prior to completing a full oscillation, dissipating energy in the process. Thus the expectation in the strong collisional regime is that the plasma may effectively absorb the dark photon field, prior to or during superradiance.

Notice that for the largest number densities and smallest velocities considered in this work, collisions become important for dark photon masses $m_{\gamma^{\prime}} \lesssim 10^{-15} \mathrm{eV}$ and initial thermal velocities $v_{e} \simeq 10^{-3} \sqrt{T /\left(10^{4} \mathrm{~K}\right)}$. However, if the electrons are accelerated by the (superradiantly grown) dark photon, the collisional rate will decrease dramatically and will become irrelevant for all dark photon masses of interest, given the strong dependence on $v_{e}$ of the Coulomb collision rate [Eq. (9)]. As we will show in a moment, the electrons will be always accelerated up to relativistic speeds on the timescales of interest. Therefore we expect collisions could play an important role only in the initial stages of the superradiant growth.

For small electron velocities (valid for the early stages of superradiance), one can drop both the magnetic field term as well as the nonlinear gradient contribution to Eq. (6), yielding an electron velocity

$$
\vec{v}_{e}=\frac{-e}{m_{e}(\nu-i \omega)}\left(\vec{E}+\sin \chi_{0} \vec{E}^{\prime}\right) .
$$

Writing the photon and hidden photon fields in Fourier modes, taking the nonrelativistic limit, and decomposing the transverse and longitudinal field components yields two coupled sets of differential equations:

$0=\left[\left(-\omega^{2}+k^{2}\right)+\left(\begin{array}{cc}\frac{\omega_{p}^{2}}{(1+i \nu / \omega)} & \frac{\sin \chi_{0} \omega_{p}^{2}}{(1+i \nu / \omega)} \\ \frac{\sin \chi_{0} \omega_{p}^{2}}{(1+i \nu / \omega)} & \frac{\sin ^{2} \chi_{0} \omega_{p}^{2}}{(1+i \nu / \omega)}+m_{\gamma^{\prime}}^{2}\end{array}\right)\right]\left(\begin{array}{c}A_{T} \\ A_{T}^{\prime}\end{array}\right)$

and

$0=\left[-\omega^{2}+\left(\begin{array}{cc}\frac{\omega_{p}^{2}}{(1+i \nu / \omega)} & \frac{\sin \chi_{i} \omega_{p}^{2}}{(1+i \nu / \omega)} \\ \frac{\sin \chi_{0} \omega_{p}^{2}}{(1+i \nu / \omega)} & \frac{\sin ^{2} \chi_{0} \omega_{p}^{2}}{(1+i \nu / \omega)}+\frac{m_{\gamma^{\prime}}^{2}}{\left(1-k^{2} / \omega^{2}\right)}\end{array}\right)\right]\left(\begin{array}{l}A_{L} \\ A_{L}^{\prime}\end{array}\right)$.

The eigenvalues and eigenvectors of the mass matrix allow us to identify the mass and composition of the propagating states. 
The limit $m_{\gamma^{\prime}} \gg \omega_{p}$ is trivial; in this case in-medium effects are not important and the eigenstates of the propagating mode with mass $m_{\gamma^{\prime}}$ coincides with $A^{\prime}$.

In the limit $m_{\gamma^{\prime}} \ll \omega_{p}$ instead, the dark photon $A_{\mathrm{dp}}$ is identified as the propagating state with mass $m_{\gamma^{\prime}}$ and is comprised of a linear combination of $A$ and $A^{\prime}$, with

$$
\begin{aligned}
& A \sim-\sin \chi_{0} A_{\mathrm{dp}}\left(1+\frac{m_{\gamma^{\prime}}{ }^{2}\left(1+i \nu / m_{\gamma^{\prime}}\right)}{\omega_{p}^{2}}\right) \\
& A^{\prime} \sim A_{\mathrm{dp}}\left(1-\frac{m_{\gamma^{\prime}} \sin ^{2} \chi_{0}\left(1+i \nu / m_{\gamma^{\prime}}\right)}{\omega_{p}^{2}}\right) .
\end{aligned}
$$

The observable electric field is then given by the combination

$$
A_{\mathrm{obs}}=A+\sin \chi_{0} A^{\prime} \sim-\sin \chi_{0} \frac{i m_{\gamma^{\prime}} \nu}{\omega_{p}^{2}} A_{\mathrm{dp}}
$$

Note that $A_{\mathrm{obs}}=0$ when $\chi_{0}=0$, since we are focusing only on the dark electromagnetic field (which we shall occasionally refer to simply as the electromagnetic field, as the SM Maxwell field is irrelevant for our analysis).

We see here that an effective in-medium suppression of the mixing proportional to $m_{\gamma^{\prime}} \nu / \omega_{p}^{2}$ arises at small masses. Physically, this happens because the plasma can efficiently move in response to the external field, oscillating in such a way so as to induce a partial cancellation.

The frequency of the dark photon field can be obtained by solving the coupled differential equations expressed in Eqs. (14) and (15), and is in general complex. In the nonrelativistic limit, the real part can be identified with the mass of the propagating state, while the imaginary part induces dissipation in the medium; this effect is akin to the concept of a skin-depth, where the imaginary part identifies the length scale over which the field drops by an $e$-fold [49]. The skin-depth of the dark photon field in the limit $m_{\gamma^{\prime}} \ll \omega_{p}$ is given by

$$
\delta_{\mathrm{dp}, \mathrm{i}} \sim\left(\nu \frac{m_{\gamma^{\prime}}^{2}}{2 \omega_{p}^{2}} \sin ^{2} \chi_{0}\right)^{-1}
$$

From this we see that the skin-depth of the dark photon is always much larger than any of the scales of interest. As a consequence, one can conclude that dissipation effects in the medium surrounding the $\mathrm{BH}$ are not sufficient to inhibit the growth of the dark photon field. Thus, the dark photon field will grow, and the (dark) electric field generated via this process will drive the electrons and ions to larger velocities until the electron-ion collision timescale has become longer than the characteristic driving frequency for all dark photon masses of interest. The immediate consequence is that the effect of collisions can be neglected. Therefore in the next section we will focus on the collisionless regime.

\section{B. Collisionless regime}

Dropping the collision term in Eq. (6) dramatically simplifies the problem at hand. In this case, one can derive a general solution for the response of the plasma to an oscillating transverse wave; we defer this derivation (generalizing previous electromagnetic solutions [62-64] to include the presence of the dark photon) to Appendix B, and only present the results here.

The mixing equation for the transverse modes in this case can be expressed as

$0=\left[-\omega^{2}+\left(\begin{array}{cc}\frac{\omega_{p}^{2}}{\gamma} & \frac{\sin \chi_{0} \omega_{p}^{2}}{\gamma} \\ \frac{\sin \chi_{0} \omega_{p}^{2}}{\gamma} & \frac{\sin ^{2} \chi_{0} \omega_{p}^{2}}{\gamma}+m^{2}\end{array}\right)\right]\left(\begin{array}{l}A_{T} \\ A_{T}^{\prime}\end{array}\right)$,

where $\gamma$ is the time-averaged boost factor characterizing the motion of the plasma. In general, the plasma motion will be determined by the field itself, making this problem difficult to solve self-consistently (this is because the field is induced by the dark photon, which is a linear combination of $A$ and $A^{\prime}$, but the appropriate weights must be determined by solving the mixing equation, which itself depends on the weights).

One can, however, identify various independent regimes in which these equations can be solved. During the initial stages of superradiance, the electric field induced by the dark photon cloud is small and the electrons are expected to be nonrelativistic. In this regime, one can take either the limit in which $m_{\gamma^{\prime}} \gg \omega_{p}$ or $\omega_{p} \gg m_{\gamma^{\prime}}$, in each case finding

$$
A_{\mathrm{obs}} \sim \begin{cases}\sin \chi_{0} A_{\mathrm{dp}} & \text { if } m_{\gamma^{\prime}} \gg \omega_{p} \\ \frac{m_{\gamma^{\prime}}^{2}}{\omega_{p}^{2}} \sin \chi_{0} A_{\mathrm{dp}} & \text { if } m_{\gamma^{\prime}} \ll \omega_{p} .\end{cases}
$$

The former of these is consistent with recovering the vacuum mixing, while the latter experiences a strong inmedium suppression. As the cloud continues to grow, the plasma may be driven to relativistic speeds. Should $\omega_{p \text {,eff }} \equiv \omega_{p} / \sqrt{\gamma} \ll m_{\gamma^{\prime}}$, one can see that Eq. (20) will reduce to the vacuum solution with $\sin \chi \sim \sin \chi_{0}$. As shown in Appendix B, the $\gamma$ factor can be expressed in terms of the applied electric field as $\gamma=\sqrt{1+\left(e E_{\mathrm{obs}} /\left(m_{e} \omega\right)\right)^{2}}$. Thus, for small dark photon masses $m_{\gamma^{\prime}}$ one must follow the evolution of the plasma boost factor as the superradiant cloud grows, assuming an observable electric field generated by the in-medium suppressed value show in Eq. (21), to determine whether the boost becomes sufficiently large so as to remove the suppression altogether. In the numerical work that follows, we implement this transition using a sharp 
TABLE I. Key parameters determining if and when dark photon superrradiance quenches. These parameters are: the kinetic mixing $\chi$ and the mass $m_{\gamma^{\prime}}$ of the dark photon, $n_{e}$ the electron number density near the $\mathrm{BH}$, the plasma frequency $\omega_{p}$, the dark photon number density when superradiance begins extracting a significant fraction of the BH spin (equivalently this can be thought of the approximate upper limit on the dark photon number density in the limit that the boson is truly noninteracting), time-averaged boost factor $\langle\gamma\rangle$, and the dark photon number density when neutron hydrogen ionizes $n_{\gamma^{\prime}}^{\text {ionization }}$.

\begin{tabular}{lcr}
\hline \hline Parameter & Description & Typical values of interest \\
\hline$\chi$ & Kinetic mixing & $10^{-8}-10^{-2}$ \\
$m_{\gamma^{\prime}}$ & Dark photon mass & $10^{-18}-10^{-10} \mathrm{eV}$ \\
$n_{e}$ & Electron density & $10^{-4}-10^{4} \mathrm{~cm}^{-3}$ \\
$\omega_{p}=\sqrt{\left(4 \pi \alpha n_{e}\right) / m_{e}}$ & Plasma frequency & $10^{-13}-10^{-9} \mathrm{eV}$ \\
$n_{\gamma^{\prime}}^{\text {sr }}$ & Saturation number density & $\lim _{\chi \rightarrow 0} 10^{57} \mathrm{~cm}^{-3} \frac{m_{\gamma^{\prime}}}{10^{-11} \mathrm{eV}}$ \\
$\langle\gamma\rangle$ & Boost factor & $1-10^{13}$ \\
$n_{\gamma^{\prime}}^{\text {ionization }}$ & Number density requited to ionize hydrogen & $10^{34} \frac{1}{\sin ^{2} \chi} \frac{10^{-11}}{m_{\gamma^{\prime}}} \mathrm{cm}^{-3}$ \\
\hline \hline
\end{tabular}

cut-off; while this is clearly naive, the transition is expected to occur rapidly and thus should represent a rough approximation to reality.

\section{Summary of in-medium suppression and effect of collisions}

To summarize, the various regimes discussed include

(i) Vacuum regime: $\nu, \omega_{p} \ll m_{\gamma^{\prime}}$. Here there are no inmedium effects. The external induced fields oscillate at a frequency larger than the natural oscillation frequency of the plasma, driving the plasma in such a way that no counterbalancing fields can be generated. Dissipative effects are strongly suppressed and can be neglected.

(ii) Collisional regime: $m_{\gamma^{\prime}} \ll \nu, \omega_{p}$. The dark photon interactions are initially suppressed by in-medium effects, and the large electron-ion collision rate induces an imaginary part of the dark photon frequency; this is equivalent to saying that the dark photon field dissipates energy continuously via inverse bremsstrahlung absorption as it propagates. For the environments of interest, this energy dissipation is never large enough to significantly suppress or absorb the dark photon field, and thus superradiance will occur and will drive $\nu \rightarrow 0$. Notice also that in general as $m_{\gamma^{\prime}} \rightarrow 0$ the dark photon fully decouples from electromagnetism.

(iii) Collision-less regime: $\nu \ll m_{\gamma^{\prime}} \ll \omega_{p}$. For small field values, the dark photon will have an in-medium suppression of the mixing. However, as the observed electric field grows, the effective plasma mass will be driven toward zero and the vacuum mixing will be recovered.

As we will show below, this in-medium suppression can be efficiently removed if the dark photon mass $m_{\gamma^{\prime}} \gtrsim$ $10^{-16} \mathrm{eV}$ and the vacuum mixing $\sin \chi_{0} \gtrsim 10^{-7}-10^{-8}$, depending on the ambient electron density.

\section{QUENCHING THE GROWTH OF THE DARK PHOTON}

In this section we outline the dominant quenching mechanisms for kinetically mixed dark photons. We show that for sufficiently large kinetic mixings, dark photon superradiance may produce time-oscillating electromagnetic signatures arising from semi-Compton scattering and synchrotron emission of the ambient electrons. In Table I we provide a glossary for a number of fundamental parameters which will control the superradiance quenching (see also Fig. 1 for an illustration of the growth and quenching process).

\section{A. Scattering processes}

The evolution and quenching of the superradiant instability in the context of the SM photon was recently studied in-depth in [30,31]; the case of the dark photon can be understood analogously with two notable exceptions. First, unlike the SM photon, the dark photon has a bare mass that will not be modified by the presence of strong electric fields or by modifications to the local electron density. Second, the dark photon scattering rate intrinsically depends on the properties of the ambient plasma, which themselves may depend on the energy density of the dark photon in a more involved way than for the SM photon. In both cases, however, one might expect the exponentially growing boson cloud to generate strong (dark) electromagnetic fields, and if these relativistic oscillations can be reached, Compton scattering (or more appropriately semi-Compton in the case of the dark photon) and synchrotron emission may produce energy losses capable of balancing the energy being extracted from the $\mathrm{BH}$ spin. The focus on what follows is on identifying the dark photon parameter space for which this occurs. It is important to note that we do not require the dark photon to be the dark matter, as the superradiant instability can be triggered for arbitrarily small abundances. 


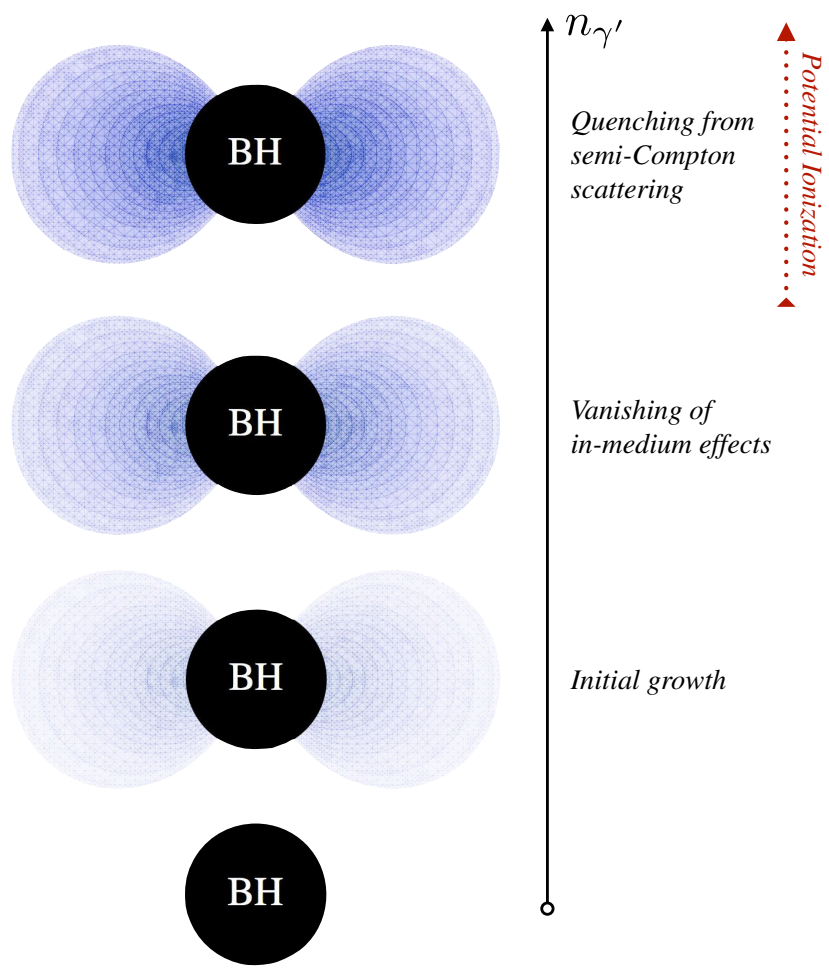

FIG. 1. Heuristic evolution illustrating the growth and quenching of the superradiant dark photon cloud. For dark photons with vacuum kinetic mixings $\chi_{0} \gtrsim \chi_{\text {min }}$ (i.e., the minimum mixing required to quench superradiance) this behavior can be characterized as follows: after an initial stage of growth, the in-medium suppression of the mixing (should it exist) is removed due to the large electric fields generated by the dark photon. At larger number densities the growth fully saturates as semi-Compton scattering removes energy at a rate equivalent to the energy injection rate from superradiance. Depending on the dark photon mass, the Stark effect may ionize all neutral hydrogen atoms in the latter stages near saturation.

Owning to the kinetic mixing, the dark photon will induce an electric force on the ambient electrons that oscillates with frequency $\omega_{R}$. Assuming the motion of the electrons to be dominated by that of the electric field (a valid assumption at large number densities), one can approximate the time-dependent boost factor of the electrons as

$$
\gamma(t)=\sqrt{1+\sin ^{2} \chi \frac{n_{\gamma^{\prime}} \cos ^{2}\left(\omega_{R} t\right)}{m_{e}^{2} m_{\gamma^{\prime}}}} .
$$

In the limit of large dark photon number density, the timeaveraged gamma factor is approximately given by $\langle\gamma\rangle \sim$ $\sin \chi \sqrt{n_{\gamma^{\prime}} / m_{\gamma^{\prime}}} / m_{e} \simeq 5.4 \times 10^{3} \sin \chi\left(\frac{n_{\gamma^{\prime}}}{10^{21} \mathrm{~cm}^{-3}}\right)^{1 / 2}\left(\frac{10^{-12} \mathrm{eV}}{m_{\gamma^{\prime}}}\right)^{1 / 2}$. In this case the energy loss from semi-Compton scattering $\gamma^{\prime}+e^{-} \leftrightarrow \gamma+e^{-}$is given by

$$
\begin{aligned}
\frac{d E_{\mathrm{SC}}}{d t} & =\frac{4}{3}\left(\gamma^{2}-1\right) \sin ^{2} \chi \sigma_{T} \rho_{\gamma^{\prime}} n_{e} \\
& \stackrel{\gamma \gg 1}{\sim} \frac{4}{3} \sin ^{4} \chi \frac{n_{\gamma^{\prime}}^{2}}{m_{e}^{2}} \sigma_{T} n_{e} .
\end{aligned}
$$

Similarly, the energy loss rate via synchrotron emission, is given by

$$
\frac{d E_{\mathrm{syn}}}{d t}=\frac{d E_{\mathrm{sC}}}{d t} \sin ^{2} \zeta
$$

where $\zeta$ is the angle between the electron velocity and the induced magnetic field. We have verified explicitly that the typical center of mass energy of the photons $\sim \gamma m_{\gamma^{\prime}}$ is never large enough that Klein-Nishina corrections become important.

\section{B. The evolution and quenching}

In order for the above processes to be capable of quenching the growth of the dark photon cloud, they must be faster than superradiant itself, which injects energy at a rate

$$
\frac{d E_{\mathrm{sr}}}{d t} \simeq 2 \frac{m_{\gamma^{\prime}} n_{\gamma^{\prime}}}{\tau_{\mathrm{sr}}}
$$

The dark-photon condensate will grow until the two energy losses compensate each other, which happens at the saturation particle density (for $m=1$, and assuming the plasma is already relativistic)

$$
\begin{aligned}
n_{\gamma^{\prime}}^{\mathrm{sat}} & \sim \frac{6 m_{e}^{2} \tilde{a}}{n_{e} \sigma_{T} \sin ^{4} \chi} m_{\gamma^{\prime}}^{2} \alpha^{6} \\
& \approx 10^{21} \mathrm{~cm}^{-3} \frac{\tilde{a}}{\sin ^{4} \chi}\left(\frac{10^{4} \mathrm{~cm}^{-3}}{n_{e}}\right)\left(\frac{m_{\gamma^{\prime}}}{10^{-12} \mathrm{eV}}\right)^{2}\left(\frac{\alpha}{0.1}\right)^{6},
\end{aligned}
$$

where $\tilde{a}$ is the initial dimensionless spin of the $\mathrm{BH}$. At saturation the field has extracted from the $\mathrm{BH}$ an amount of energy given by

$$
M_{\text {cloud }}=m_{\gamma^{\prime}} n_{\gamma^{\prime}}^{\text {sat }} \mathcal{V}
$$

where $\mathcal{V}=\frac{4 \pi}{3} r_{\text {cloud }}^{3}$ is the volume of the cloud. For a single azimuthal mode (typically $m=1$, see Ref. [65] for the case of multiple modes), any energy extraction $\delta M$ is proportional to the angular momentum extraction $\delta J$ through $\delta M=\omega_{R} / m \delta J[7]$.

In order to evade current bounds coming from gaps in the $\mathrm{BH}$ mass-spin plane or from gravitational-wave emission, we require that quenching is sufficiently efficient so that it would allow for extraction of a negligible amount of angular momentum, i.e., $\delta J / J \ll 1$. Using the equations above, this is equivalent to 


$$
\sin ^{4} \chi \gg \frac{8 \pi m_{e}^{2}}{n_{e} \sigma_{T}} m_{\gamma^{\prime}} \alpha
$$

Note that, as previously discussed, $\chi$ is the in-medium coupling which depends on various parameters of the system, including the number density of dark photons (which controls whether the in-medium suppression has been removed). In the next section we shall use this condition to identify the parameter space in the $\sin \chi_{0}-$ $m_{\gamma^{\prime}}$ plane where the superradiant instability is efficiently quenched; we heuristically outline the growth and saturation of the cloud for a generic dark photon model in Fig. 1.

In order to determine whether a given dark photon model, defined by mass and vacuum mixing angle, quenches before extracting sufficient $\mathrm{BH}$ spin, one must first define both the $\mathrm{BH}$ mass of interest, or equivalently the superradiant coupling $\alpha \equiv m_{\gamma^{\prime}} M$, and the local electron number density. The former is responsible for setting the superradiant timescale, and the latter controls the efficiency of the semi-Compton and synchrotron emission. In order to be conservative, we adopt a fiducial value of $\alpha=0.4$, for which $\tau_{\text {sr }}$ of a near-extremal $\mathrm{BH}$ is maximal, however comparable values of $\alpha$ do not yield significantly different results. The range of $n_{e}$ expected in the vicinity of a $\mathrm{BH}$ is estimated in Appendix A and may span many orders of magnitude. Rather than adopting a particular value, in what follows we show results under various assumptions in order to be as broad as possible.

A final word is in order regarding the electron number density. The ambient gas around BHs after reionization or in environments with large thermal accretion rates is expected to be largely ionized. This may not necessarily be the case however, implying the free electron number density may be suppressed relative to that of neutral hydrogen. This opens the possibility that the neutral hydrogen can be ionized during the superradiant growth, further increasingly the electron number density, and subsequently the scattering rate. Ionization from the growing dark photons can occur via the Stark effect [31], in which the induced electric field becomes large enough to shift the ground state of neutral hydrogen from $13.6 \mathrm{eV} \rightarrow 0 \mathrm{eV}$. Following [31], the number density of dark photons required to ionize the hydrogen is

$$
n_{\gamma^{\prime}}>n_{\gamma^{\prime}}^{\text {ionization }} \sim 10^{35} \frac{1}{\sin ^{2} \chi}\left(\frac{10^{-12} \mathrm{eV}}{m_{\gamma^{\prime}}}\right) \mathrm{cm}^{-3},
$$

which should be compared to Eq. (26), suggesting this may happen when

$\sin ^{2} \chi \lesssim 2 \times 10^{-14} \tilde{a}\left(\frac{10^{4} \mathrm{~cm}^{-3}}{n_{e}}\right)\left(\frac{m_{\gamma^{\prime}}}{10^{-12} \mathrm{eV}}\right)^{3}\left(\frac{\alpha}{0.1}\right)^{6}$

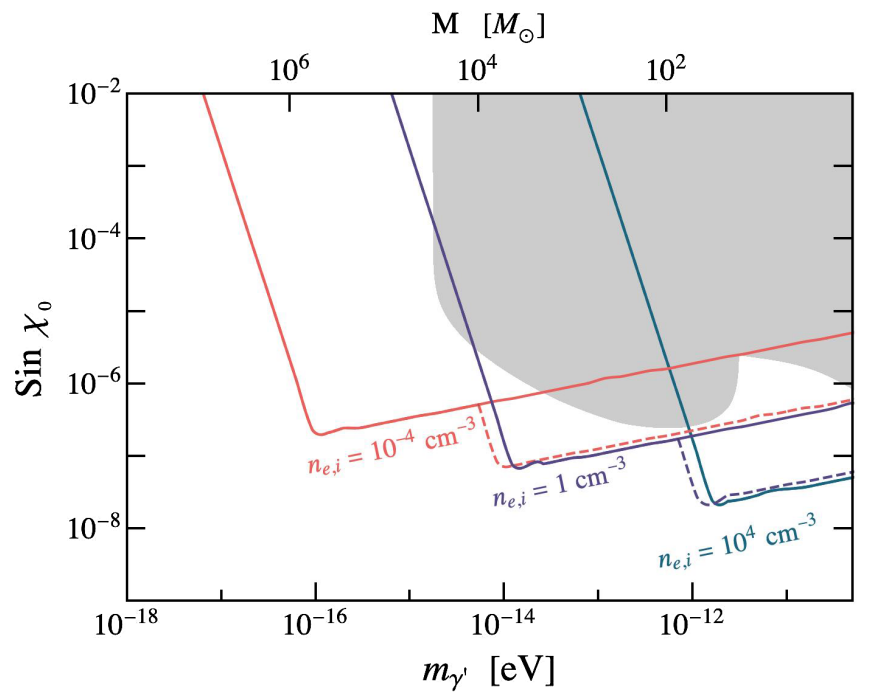

FIG. 2. Lower bound on kinetic mixing $\chi_{0}$ for which quenching of superradiance occurs, assuming local electron number densities of $10^{-4}, 1$, and $10^{4} \mathrm{~cm}^{-3}$ and $\alpha=0.4$. Short dashed lines account for the possibility that the Stark effect could increase the local electron number density if the initial medium is not fully ionized (see text for details; $x_{e}$ taken to be $2 \times 10^{-4}$ ). The approximate range of $\mathrm{BH}$ masses being probed is shown on the upper $\mathrm{x}$-axis, and is derived assuming $\alpha=0.4$. Kinetic mixings below the values shown here may still be capable of spinning down massive BHs, depending on additional details of the model. The gray region corresponds to current constraints on dark photons, derived from the cosmic microwave background $[66,67]$.

The above equation assumes $n_{\gamma^{\prime}}^{\text {ionization }}$ is still sufficiently low so that the black hole has not yet been spun down.

The minimum vacuum kinetic mixing $\chi_{0}$ capable of quenching superradiance at each dark photon mass is shown in Fig. 2 along with current constraints on dark photons, derived from the cosmic microwave background $[66,67]$ (note that these constraints are derived assuming dark photons do not constitute dark matter; in the case that they do comprise a non-negligible fraction, the constraints are many orders of magnitude stronger [67-69]). Here, we show results for $\alpha=0.4$ and $n_{e}=10^{-4} \mathrm{~cm}^{-3}$ (red), $1 \mathrm{~cm}^{-3}$ (purple), and $10^{4} \mathrm{~cm}^{-3}$ (blue). We also show the impact of adopting a local free electron fraction $x_{e} \equiv$ $n_{e} / n_{H}=2 \times 10^{-4}$ (consistent with the mean value of the Universe prior to reionization) - at larger masses, the local hydrogen can ionize via the Stark effect and superradiance can be quenched for smaller kinetic mixings. The reduced sensitivity at low masses arises because the in-medium suppression of the kinetic mixing cannot be removed.

The overall physical trend is manifest: an increase in the electron number density can be compensated for by adopting a smaller mixing angle. We also note that a smaller superradiance parameter $\alpha$ would imply a smaller growth rate for the superradiant cloud $\frac{d E_{\text {sr }}}{d t}$, thus allowing 

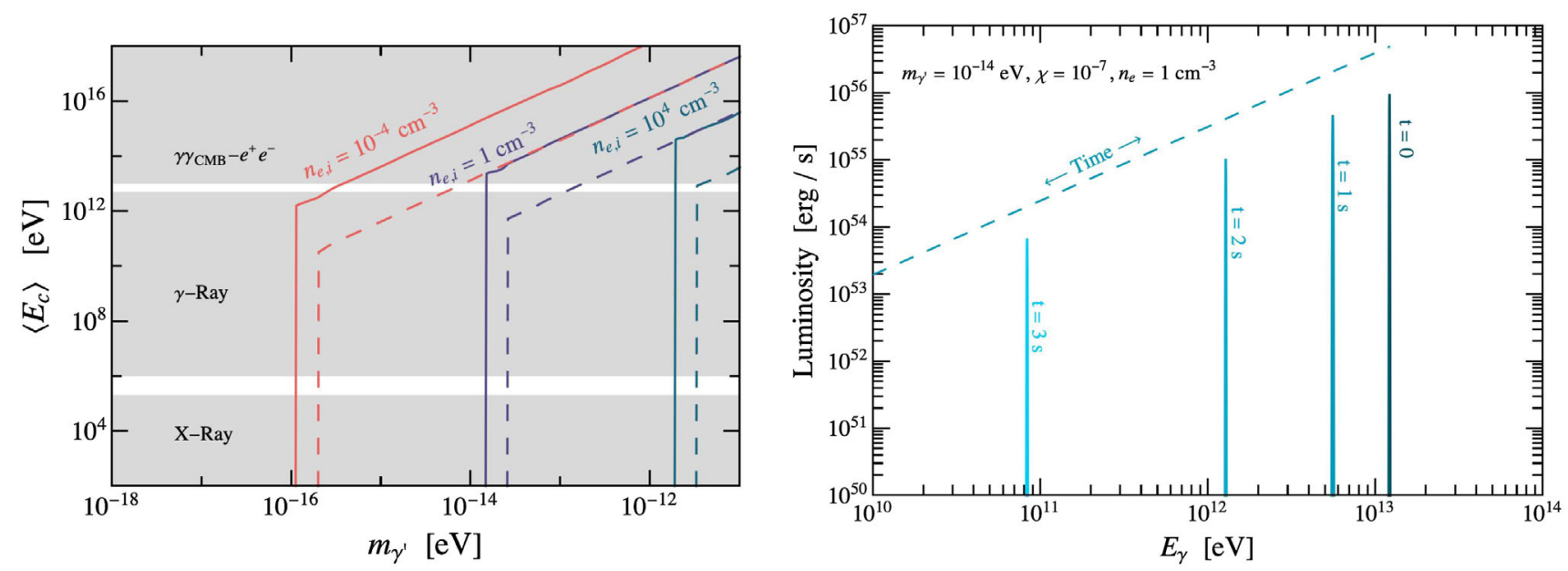

FIG. 3. Left: characteristic time averaged energy $\left\langle E_{c}\right\rangle$ of up-scattered photons, given by $\gamma^{2} m_{\gamma}$, as a function of dark photon mass, assuming various values of the local electron number density and for $M m_{\gamma^{\prime}}=0.4$. Solid lines take $\chi_{0}$ to be the minimum value capable of quenching superradiance $\chi_{\min }$, while dashed blue line takes $\chi_{0}=10 \chi_{\min }$ for one representative case. Approximate energy ranges over which telescopes observe $\gamma$-rays, $x$-rays, and the CMB are highlighted. We also highlight the regime where $\gamma$-rays can scatter of CMB photons and pair produce $e^{+} e^{-}$; this process may generate TeV halos in a similar mechanism to blazars. Right: time evolution of the electromagnetic signal for $m_{\gamma^{\prime}}=10^{-14} \mathrm{eV}, \sin \chi_{0}=10^{-7}$, and $M m_{\gamma^{\prime}}=0.4$.

smaller scattering rates (achieved e.g., by lowering the mixing angle) to induce quenching. Low dark photon masses are notoriously difficult to probe due to the strong in-medium suppression; nevertheless, it seems quenching can be achieved to some degree for masses as small as $\sim 10^{-17} \mathrm{eV}$.

\section{Observable signatures}

We have shown in the previous section that dark photons with vacuum mixings $\chi_{0} \gtrsim 10^{-8}$, depending on the mass, can quench superradiance prior to the spin down of the $\mathrm{BH}$. This quenching is a consequence of the fact that the accelerated electrons radiate synchrotron emission and semi-Compton scatter off the ambient dark photon. These processes result in the direct emission of photons, with the characteristic energy $\left\langle E_{c}\right\rangle$ of each processes roughly given by the inverse semi-Compton formulas $\sim \gamma^{2} \omega_{g}$ and $\sim \gamma^{2} m_{\gamma^{\prime}}$, respectively, where $\omega_{g}=e B /\left(m_{e} \gamma\right)$ is the gyro frequency.

The luminosity of each process is roughly given by $\mathcal{V} \times$ $d E / d t$ at the saturation density (26). Assuming $\chi$ is sufficiently large to saturate the growth, the maximum luminosity and characteristic energy for the semi-Compton process then yields

$$
\begin{aligned}
L_{\mathrm{sC}}^{\max } \simeq & 3 \times 10^{58} \frac{\mathrm{erg}}{\mathrm{s}} \tilde{a}^{2}\left(\frac{m_{\gamma^{\prime}}}{10^{-12} \mathrm{eV}}\right)\left(\frac{\alpha}{0.4}\right)^{9} \\
& \times\left(\frac{10^{-8}}{\sin \chi}\right)^{4}\left(\frac{10^{4} \mathrm{~cm}^{-3}}{n_{e}}\right),
\end{aligned}
$$

$$
\begin{aligned}
E_{\mathrm{sC}}^{\max } \simeq & 2.1 \times 10^{15} \mathrm{eV} \tilde{a}\left(\frac{\alpha}{0.4}\right)^{6} \times\left(\frac{10^{-8}}{\sin \chi}\right)^{2} \\
& \times\left(\frac{10^{4} \mathrm{~cm}^{-3}}{n_{e}}\right)\left(\frac{m_{\gamma^{\prime}}}{10^{-12} \mathrm{eV}}\right)^{2},
\end{aligned}
$$

while in the case of synchrotron emission one finds

$$
\begin{gathered}
L_{\mathrm{syn}}^{\max } \simeq \sin ^{2} \xi L_{\mathrm{sC}}^{\max }, \\
E_{\mathrm{syn}}^{\max } \simeq \gamma \frac{e B}{m_{e}} \simeq \frac{\sin \chi e}{m_{e}^{2}} n_{\gamma^{\prime}} .
\end{gathered}
$$

As expected, these are comparable processes, with semiCompton dominating due to the lack of dependence on orientation. We also stress that Eqs. (31)-(34) are valid only if superradiance is quenched. In particular, the maximum luminosity reached can never exceed the upper limit set by the physical scales in the problem $\sim M / \tau_{s}$, which is always well below Planckian luminosities [70].

We illustrate the dependence of the characteristic energy of the semi-Compton up-scattered photon $\left\langle E_{c}\right\rangle$ (left) and time-averaged luminosity arising from the time-dependent in the gamma factor (right) in Fig. 3. Results are shown by either fixing the kinetic mixing to the minimal value $\chi_{\text {min }}$ required for quenching (solid), or by taking $\chi_{0}=10 \times \chi_{\text {min }}$ (dashed), and for various electron number densities. The luminosity is maximal for the smallest kinetic mixings (because the latter correspond to higher saturation density of the dark-photon cloud), reaching values near $\sim 10^{57} \mathrm{erg} / \mathrm{s}$, well above the luminosities of, e.g., typical 
AGN. We have also highlighted in Fig. 3 various parts of the electromagnetic spectrum where this signal may be easily detectable, as well as the approximate threshold where $e^{+} e^{-}$pair production may become important (see text below). Intriguingly, because the electron boost factors vary with time (due to the oscillating electric fields), both $E_{c}$ and the luminosity have a characteristic oscillatory behavior; this is roughly illustrated in the right panel of Fig. 3, where we show the evolution over part of a period of oscillation for a particular dark photon candidate. Notice in this figure we have drawn the fixed time emission as a narrow line (importantly, this is an illustration, not a calculation); this is because both the dark photon and the plasma have narrow distributions in energy, the former being nonrelativistic and the latter being driven by the electric field. The line width is likely generated by nonlinearities in the dark photon field; while it is of great interest to understand the spectral characteristics, we consider determining this feature to be beyond the scope of this work.

Given that these (ordinary) photons are produced near the $\mathrm{BH}$ itself, a logical question to ask is whether they may escape their local environment. Assuming the local gas is ionized, the dominant interaction for photons with energies $E \lesssim 10^{7} \mathrm{eV}$ is again that of Compton scattering. In this case, the optical depth can be calculated via

$$
\tau=\int d \ell n_{e} \sigma_{T}
$$

If we parameterize the electron number density with a Bondi accretion profile, i.e., $n_{e} \propto r^{-3 / 2}$ (see Appendix A), this integral is dominated by the highest densities near the $\mathrm{BH}$ itself. Approximating the lower limit of integration as the Schwarzschild $r_{s}$, we can write the optical depth as

$$
\tau \sim 10^{-7}\left(\frac{M}{10^{8} M_{\odot}}\right)\left(\frac{n_{e, c}}{10^{4} \mathrm{~cm}^{-3}}\right)
$$

where $n_{e, c}$ is the central electron number density at $r_{s}$.

For higher energy gamma rays, the optical depth will be set either by the possibility for photons to pair produce $e^{+} e^{-}$by scattering off the extragalactic background light (EBL) or via $\gamma+p \rightarrow p+e^{+}+e^{-}$. The cross section for the former is given by [71-73]

$\sigma_{\gamma \gamma}=\frac{3 \sigma_{T}}{16}\left(1-\beta^{2}\right)\left(2 \beta\left(\beta^{2}-2\right)+\left(3-\beta^{4}\right) \log \left(\frac{1+\beta}{1-\beta}\right)\right)$,

where $\beta=\sqrt{1-4 m_{e}^{2} / s}$, and $s=2 E_{\gamma} E_{\gamma_{\mathrm{EBL}}}(1-\cos \theta)$. The mean free path for this can be expressed roughly as [74]

$$
\lambda_{\gamma \gamma} \sim 80\left(\frac{E_{\gamma}}{10 \mathrm{TeV}}\right)^{-1} \mathrm{Mpc} .
$$

The scattering off ambient protons has a cross section [72]

$$
\sigma_{\gamma p}=\alpha r_{0}^{2}\left[\frac{28}{9} \log \left(\frac{2 E_{\gamma}}{m_{e}}\right)-\frac{218}{27}\right],
$$

where $r_{0}$ is the classical electron radius. Again assuming a fully ionized medium and the radial taken from Bondi accretion, one can compute the optical depth; we find this the optical depth for this process is always quite small and can be neglected. Thus, depending on the distance to the $\mathrm{BH}$ of interest, the $\gamma \gamma$ scattering rate may be sufficient to absorb the high energy photons. In this case, the electrons produced could scatter off ambient light and generate $\gamma$-ray halos around the $\mathrm{BH}$ in a similar manner to that of blazars [74]. The detailed observational signatures are certainly worth investigating, and something we leave to future work.

\section{Comments on UV completions}

In the previous section we adopted a phenomenological approach and simply assumed that a dark photon with a given mass and kinetic mixing exists. It is however interesting to understand whether the quenching mechanism can depend on the origin of the dark photon mass. One can generically consider two different scenarios: either the mass is generated via the Stückelberg mechanism, which can be obtained from the Proca Lagrangian by introducing a fictitious gauge symmetry [75], or from the Higgs mechanism with a new scalar field $\Phi$ acquiring a vacuum expectation value $\langle\Phi\rangle=v / \sqrt{2}$.

The case in which the dark photon mass is generated via the Stückelberg mechanism leads to no novel quenching mechanisms, as no new interactions have been introduced. It is worth mentioning, however, that the so-called swampland conjecture, which is an attempt at identifying the set of effective field theories consistent with a theory of quantum gravity, presents a theoretical challenge for light dark photons with masses generated in this way [76]. Specifically, it has been argued that, for a given mass and mixing, one can compute the cut-off of the effective theory-in order for this theory to remain meaningful, the cut-off scale should likely be $\gtrsim \mathcal{O}(1) \mathrm{TeV}$, implying kinetic mixings much stronger than those considered here.

In the case of the Higgs mechanism the situation may be substantially different [28]. Consider the following Lagrangian

$$
\mathcal{L}=-\frac{1}{4} F_{\mu \nu}^{\prime} F^{\prime \mu \nu}+\frac{1}{2}\left|D_{\mu} \Phi\right|^{2}-V(\Phi),
$$

with $D_{\mu}=\partial_{\mu}-i g A_{\mu}^{\prime}$ being the covariant derivative and $\Phi$ being the generic scalar field which is then assumed to 
acquire a vev, so that in the unitary gauge the field may be expanded as $\Phi=(v+\sigma) / \sqrt{2}$. In order to be concrete, we consider here a potential of the form $V(\Phi)=\lambda\left(|\Phi|^{2}-v^{2} / 2\right)^{2}$.

After symmetry breaking, the dark photon acquires a mass $m_{A^{\prime}}=g v$; the mass of the scalar field can also be read from the potential as $m_{\sigma}=\sqrt{2 \lambda} v$. Notice that when the vector field is amplified around the rotating $\mathrm{BH}$, the scalar field picks an extra term due to the large densities $V(\Phi) \supset \frac{1}{2} g^{2}(v+\sigma)^{2} \times\left\langle A_{\mu} A^{\mu}\right\rangle$. One can check that in this case if $\left\langle A_{\mu} A^{\mu}\right\rangle>\frac{\lambda v^{2}}{g^{2}}$, the original symmetry is restored, and subsequently the dark photons become massless and can free stream away from the $\mathrm{BH}$. However, unless one chooses extremely small values of the scalar $\mathrm{vev}^{3}$ and self-interaction $\lambda$, the symmetry restoration scale is never achieved for the dark photon masses considered. It is also worth mentioning that the operator $|\Phi|^{2}$ would also couple to any neutral operator built out of SM fields-should the new vev be too low, one may also have to fine tune these additional couplings around zero to be consistent with current observations.

Finally, it is worth mentioning that the existence of any light particle in a theory directly coupled to the dark sector will allow for Schwinger pair production at sufficiently high densities [78]. This may be particularly relevant for models in which the dark photon serves as a mediator for other dark fermions playing the role, for example, of dark matter [79-81].

In the case of the SM photon, the Schwinger pair production rate is given by [82]

$$
\Gamma_{\mathrm{Schw}} \simeq \frac{m_{e}^{4}}{4 \pi^{3}}\left(\frac{\mathcal{E}}{\mathcal{E}_{c}}\right) \sum_{b=1}^{\infty} \frac{1}{b^{2}} e^{-b \pi^{\mathcal{E}_{\mathcal{C}}}},
$$

where $\mathcal{E}_{c}=m_{e}^{2} / \sqrt{4 \pi \alpha}$ and $\mathcal{E}$ is the electric field strength. Analogously, given a dark sector fermion with mass $m_{d}$-charged under the $U(1)_{\text {dark }}$ - with Lagrangian $\mathcal{L}_{\text {dark }} \supset \lambda_{d} \bar{\psi}_{d} \gamma^{\mu} \psi_{d} A_{\mu}^{\prime}$, the exponential suppression in the particle production rate will be removed when the superradiant cloud has reached a number density

$$
n_{\gamma^{\prime}}^{\text {Schw }} \sim \frac{\pi^{2} m_{d}^{4}}{\lambda_{d} m_{\gamma^{\prime}}} .
$$

Comparing this value to the maximum achievable number density by superradiance, one finds that in order to quench

\footnotetext{
${ }^{3}$ Note that for very small dark photon and dark Higgs masses the semi-Compton scattering will not be suppressed by inmedium effect, as in this regime the dark photon would couple to SM fermion as in a millicharge model $[58,77]$. However, the interaction will anyway be likely suppressed by the smallness of the required dark gauge coupling.
}

superradiance the mass of the dark sector particle must satisfy

$$
m_{d} \lesssim 6.7 \times 10^{6}\left(\frac{\lambda_{d}}{0.1}\right)^{1 / 4}\left(\frac{m_{\gamma^{\prime}}}{10^{-12} \mathrm{eV}}\right)^{1 / 2}\left(\frac{\alpha}{0.4}\right)^{5 / 4} \mathrm{eV} .
$$

\section{A COMMENT ON THE SM PHOTON MASS}

Despite the fact that the SM photon is generically considered to be massless, there still exists the possibility that it carries a nonzero bare mass; if this is the case, it could shed light on many fundamental puzzles such as why charge is quantized and the potential existence of charged BHs [83]. Additionally, in the contest of bosonic strings, the condensation of tensor fields generates a violation of Lorentz symmetry and may dress the photon with a small mass $[84,85]$. In view of these intriguing theoretical possibilities, it is important to robustly constrain the magnitude of the photon mass. Typical probes to date involve: laboratory experiments using high-frequency tests of Coulomb's law [86], study of the sector structure of the Solar wind in the presence of a finite photon mass $[87,88]$, and frequency-dependent dispersion in fast radio bursts (FRB) [89]. In fact, if the photon has a bare mass $m_{\gamma}$, it may trigger a superradiant instability in the same way as the dark photon, and the observation of highly spinning supermassive BHs has been used as an argument to constrain bare photon masses above $\sim 10^{-20} \mathrm{eV}$ [51].

It is important however to stress that the propagating mode, exciting the superradiance instability, will have an energy gap in the dispersion relation coming from both the Proca mass and the plasma contribution. Therefore, for low photon masses $m_{\gamma} \lesssim 10^{-13} \mathrm{eV}$ (note that current observations of FRBs constrain bare mass contributions above this level [89]), the plasma contribution will always be the dominant one, even for low density environments, see Eq. (10). This means that for massive BHs $\left(M \gtrsim 10^{4} M_{\odot}\right)$ superradiance will be exponentially suppressed because the effective coupling $M \omega_{p} \gg 1$ [7]. While it may be possible for environmental factors (e.g., due to dynamical or geometrical effects [30,59]) to suppress the contribution to the effective photon mass, even if superradiance could be triggered, Compton scattering will be efficient in quenching superradiance long before extracting a significant fraction of energy [31] (notice that the results presented in Fig. 2 for the dark photon can be applied to the SM photon with a bare mass in the limit $n_{e} \rightarrow 0$ and $\sin \chi_{0} \rightarrow 1$ ); as such, superradiance cannot be used to probe the bare photon mass, should it exist.

\section{CONCLUSION}

In this work we have investigated the validity of superradiant bounds on dark photons, and argued there exists large regions of parameter space in which the dark photon 
interactions quench the superradiant growth before any observable change to the $\mathrm{BH}$ spin has occurred; specifically, we have shown that this occurs for vacuum kinetic mixings typically on the order of $\chi_{0} \gtrsim 10^{-8}$, however this value depends on the mass of dark photon, the mass of the $\mathrm{BH}$, and the local electron number density. Additionally, we comment on other model-dependent quenching mechanisms, such as the minimum fermion mass required for a quenching via the Schwinger mechanism, or the restoration of the gauge symmetry which could arise in models where the dark photon arises from the Higgs mechanism. While such mechanisms are interesting in their own right, they require additional model assumptions beyond the simple assumption of kinetic mixing made here.

While dark photons undergoing premature quenching of the superradiant growth will likely leave no discernible imprint on the $\mathrm{BH}$ spin distribution, we find that such particles may be capable of generating enormous bursts of light, with time-averaged luminosities extending up to $\sim 10^{57} \mathrm{erg} / \mathrm{s}$. This signal should appear as a time- and energy-oscillating line, which could provide a striking signature of this phenomenon. This provides an alternative observational strategy for identifying ultra light bosons using $\mathrm{BH}$ superradiance.

The role of particle interactions in superradiant growth offers a rich and largely unexplored phenomenology. This work has focused on a single well-motivated extension of the SM, and showed that large regions of parameter space naturally evade conventional superradiant constraints, opening up new opportunities for detection. We hope that this will serve as a future guide toward understanding novel signatures of BH superradiance.

\section{ACKNOWLEDGMENTS}

We thank Aditya Parikh, Georg Raffelt, Toni Riotto, and Giuseppe Rossi for useful discussions. We also thank Edoardo Vitagliano and Enrico Cannizzaro for discussions and comments on the manuscript. A.C. acknowledges support from the Israel Science Foundation (Grant No. 1302/19), the US-Israeli BSF (Grant No. 2018236) and the German Israeli GIF (Grant No. I-2524-303.7). A.C acknowledges also hospitality and support from the Max Planck Institute for Physics of Munich. S. J. W. acknowledges funding from the European Research Council (ERC) under the European Union's Horizon 2020 research and innovation programme (Grant Agreement No. 864035UnDark). P. P. acknowledges financial support provided under the European Union's H2020 ERC, Starting Grant Agreement No. DarkGRA-757480. We also acknowledge support under the MIUR PRIN and FARE programmes (GW-NEXT, CUP: B84I20000100001), and from the Amaldi Research Center funded by the MIUR program "Dipartimento di Eccellenza" (CUP: B81I18001170001).

A. C. and S. J. W. contributed equally to this work.

\section{APPENDIX A: PLASMA FREQUENCY AROUND BHS}

In this Appendix we estimate the typical plasma density expected in the surrounding of an astrophysical $\mathrm{BH}$. We use a simple Bondi-Hoyle model for spherical accretion onto a $\mathrm{BH}$ moving relative to a background baryonic density,

$$
\frac{d M}{d t}=4 \pi \lambda \rho_{\infty} v_{\mathrm{eff}} r_{B}^{2},
$$

where $\lambda=\mathcal{O}(1)$ is a model-dependent factor that accounts for the nongravitational suppression of accretion, $\rho_{\infty}$ is the gas density far from the $\mathrm{BH}, v_{\text {eff }}=\sqrt{c_{\infty}^{2}+v_{\text {rel }}^{2}}$, where $c_{\infty}$ and $v_{\text {rel }}$ are the speed of sound and the relative BH-baryon velocity far from the $\mathrm{BH}$, respectively, and

$$
r_{B}=\frac{M}{v_{\mathrm{eff}}^{2}}
$$

is the Bondi radius. For typical nonrelativistic effective velocities, the Bondi radius is much bigger than the $\mathrm{BH}$ gravitational radius, i.e., $r_{B} \gg M$. In this regime the total infalling flow of baryons toward the $\mathrm{BH}$ is roughly constant, i.e.,

$$
n_{e}(r) v_{\mathrm{ff}}(r) r^{2}=\mathrm{const},
$$

where $v_{\mathrm{ff}}(r)=\sqrt{v_{\mathrm{eff}}^{2}+\frac{2 M}{r}-\frac{2 M}{r_{B}}}$ is the free fall velocity of the gas, which reduces to $v_{\text {eff }}$ at the Bondi radius. Therefore, the electron density profile within the Bondi sphere reads

$$
n_{e}(r)=n_{e}^{\infty} \frac{v_{\mathrm{eff}} r_{B}^{2}}{v_{\mathrm{ff}}(r) r^{2}}
$$

where $n_{e}^{\infty}=\rho_{\infty} / m_{e}$. At distances $r \sim \mathcal{O}(M), v_{\mathrm{ff}}(r) \sim$ $\sqrt{2 M / r} \gg v_{\text {eff }}$, so that

$$
n_{e}(r) \sim n_{e}^{\infty} \frac{v_{\mathrm{eff}}}{\sqrt{2 M}} \frac{r_{B}^{2}}{r^{3 / 2}}
$$

Finally, near the peak of the BH-boson condensate, $r_{\text {cloud }} \sim M / \alpha^{2}$, we get

$n_{e}\left(r_{\text {cloud }}\right) \sim \frac{n_{e}^{\infty}}{\sqrt{2} v_{\text {eff }}^{3}} \alpha^{3} \approx 7 \times 10^{2} n_{e}^{\infty}\left(\frac{\alpha}{0.1}\right)^{3}\left(\frac{0.01}{v_{\text {eff }}}\right)^{3}$,

which is valid provided $r_{\text {cloud }} \lesssim r_{B}$, i.e., $\alpha \gtrsim v_{\text {eff }}$. The above estimate shows that the typical electron density in the relevant region near the $\mathrm{BH}$ can be several orders of magnitude larger than the "ambient" density at infinity, $n_{e}^{\infty}$, depending on $\alpha$ and $v_{\text {eff }}$. Correspondingly, the plasma density $\omega_{p} \propto n_{e}^{1 / 2}$ can also vary by some orders of 
magnitude, see Eq. (10). In the main text we keep the local density $n_{e}$ near the $\mathrm{BH}$ as a free parameter in the range $n_{e} \in\left(10^{-4}, 10^{4}\right) \mathrm{cm}^{-3}$, which should bracket the model uncertainties.

\section{APPENDIX B: NONLINEAR REGIME}

In this appendix we rederive the results of Refs. [62,63], extending them to the electrodynamics with a massive dark photon. We can write Maxwell equations for both SM photon:

$$
\begin{gathered}
\nabla \times \vec{E}=-\partial \vec{B} / \partial t, \\
\nabla \cdot \vec{B}=0, \\
\nabla \times \vec{B}=4 \pi n_{e} e \vec{v}_{e}+-\partial \vec{E} / \partial t, \\
\nabla \cdot \vec{E}=4 \pi e n_{e},
\end{gathered}
$$

and for a dark electromagnetic field:

$$
\begin{gathered}
\nabla \times \vec{E}^{\prime}=-\partial \overrightarrow{B^{\prime}} / \partial t \\
\nabla \cdot \overrightarrow{B^{\prime}}=0 \\
\nabla \times \overrightarrow{B^{\prime}}=4 \pi n_{e} e \vec{v}_{e}+-\partial \vec{E}^{\prime} / \partial t-m_{\gamma^{\prime}}^{2} \vec{A}^{\prime} \\
\nabla \cdot \vec{E}=4 \pi e n_{e}-m_{\gamma^{\prime}}^{2} A^{\prime 0}
\end{gathered}
$$

These equations should be considered together with the electron equations of motion Eq. (7), ignoring the collision term which we have seen to be not important for our goals. We want to study the generic problem of wave motions of the plasma, we therefore define the variable $\xi=\vec{i} \cdot \vec{r}-U t$, where $\vec{i}$ is a constant unit vector and $U$ the phase velocity of the wave. Using this new variable the equation of motion for the electrons becomes

$$
\left(\vec{i} \cdot \vec{v}_{e}-U\right) \partial \vec{p}_{e} / \partial_{\xi}=e \vec{E}_{\mathrm{obs}}+e \vec{u}_{e} \times \vec{B}_{\mathrm{obs}},
$$

where we defined the observable fields $\vec{B}_{\text {obs }}=\vec{B}+\sin \chi_{0} \vec{B}^{\prime}$, $\vec{E}_{\text {obs }}=\vec{E}+\sin \chi_{0} \vec{E}^{\prime}$.

Now take the vector product of this equation with the wave vector $\vec{i}$ and consider only transverse waves $\left(\vec{i} \cdot \vec{B}_{\text {obs }}=0\right)$ to get

$$
\left(\vec{i} \cdot \vec{v}_{e}-U\right) \vec{i} \times \dot{\vec{p}}_{e}=e \vec{i} \times \vec{E}_{\mathrm{obs}}-e \vec{i} \cdot \vec{v}_{e} \vec{B}_{\mathrm{obs}},
$$

where the overdot indicates a derivative respect to the wave variable $\xi$. Using Faraday's law, we can also derive

$$
\vec{i} \times \vec{E}_{\mathrm{obs}}=U \vec{B}_{\mathrm{obs}},
$$

where we neglected background magnetic fields, as we are interested in a plasma which performs self-oscillations. We then consider Ampere's law, multiplied vectorially on the left by $\vec{i}$ to obtain

$$
\dot{\vec{B}}_{\mathrm{obs}}=\frac{4 \pi\left(1+\sin \chi_{0}^{2}\right) e n_{e}}{\left(U^{2}-1\right)} \vec{i} \times \vec{v}_{e}-\frac{\sin \chi_{0} m_{\gamma^{\prime}}^{2}}{U^{2}-1} \vec{i} \times \vec{A}^{\prime},
$$

where the dark vector potential can be related to the dark magnetic field via $\vec{i} \times \dot{\vec{A}}^{\prime}=\vec{B}^{\prime}$. This latter term is important in the limit of $m_{\gamma^{\prime}} \gg \omega_{p}$, when the propagating light mode state - triggering superradiance - is aligned with $A^{\prime}$ and we can write $\vec{A}^{\prime} \simeq-1 /\left(e \sin \chi_{0}\right) \vec{p}_{e}$. Consequently, we can take the derivative of Eq. (B10) to arrive to a second order differential equation for the electron momentum. In order to directly compare with Refs. [62,63] let us rescale our variables, defining $\vec{\rho}=\vec{p}_{e} / m_{e}$ and rescaling the wave variable $\xi \rightarrow \xi / \omega_{p}$. We then get

$\frac{d^{2} \vec{\rho}}{d \xi^{2}}+\frac{\vec{\rho}}{\left(\beta^{2}-1\right) \sqrt{1+\rho^{2}}}\left(1+\frac{m_{\gamma^{\prime}}^{2}}{\omega_{p}^{2}} \sqrt{1+\rho^{2}}\right)=0$,

which is our master equation. The first term in the brackets is the one relevant when $m_{\gamma^{\prime}} \ll \omega_{p}$, while the second one should be considered only in the regime $m_{\gamma^{\prime}} \gg \omega_{p}$.

Without loss of generality we can limit to consider the simple case where $\vec{i}=\hat{z}$, obtaining the solutions

$$
\begin{aligned}
& \rho_{x}=\rho \cos (\omega(t-z / U)), \\
& \rho_{y}=\rho \sin (\omega(t-z / U)) .
\end{aligned}
$$

The frequency of oscillations is different depending on the hierarchy between $m_{\gamma^{\prime}}$ and $\omega_{p}$. When $\omega_{p} \gg m_{\gamma^{\prime}}$, we find a dielectric function $\epsilon(\omega) \equiv 1 / U^{2}$ given by

$$
\epsilon(\omega) \simeq 1-\omega_{p}^{2}\left(1+\rho^{2}\right)^{-1 / 2} / \omega^{2} .
$$

If instead $\omega_{p l} \ll m_{\gamma^{\prime}}$ to start with, the shortest timescale in the problem is always dictated by the oscillation of the dark fields and the dielectric function then simply reads

$$
\epsilon(\omega) \simeq 1-m_{\gamma^{\prime}}^{2} / \omega^{2} .
$$

The modulus $\rho$ can be connected to the observed magnetic and electric field using $\vec{i} \times \dot{\vec{p}}=-e \vec{B}_{\mathrm{obs}}$ and $\vec{B}=1 / U \vec{i} \times \vec{E}$. We then find

$$
\rho^{2}=e^{2} E_{\mathrm{obs}}^{2} / m_{e}^{2} \omega^{2} .
$$

We notice now that by definition the velocity of the electrons is $u_{e}^{i}=\rho^{i} / \gamma$, where $\gamma$ is the boost factor. We can then plug this solution for the electron momentum back 
into the spatial current term of Proca equations Eq. (8). Working in the non relativistic limit, we find the mass matrix in Eq. (20), where the boost factor is a timeaveraged one, due to the sinusoidal functions in
Eq. (B15). A large boost factor for the electrons lower the plasma frequency as in the SM case. When the electrons move very fast, it will be very hard to have and excite any collective mode.
[1] W. H. Press, Astrophys. J. 170, L105 (1971).

[2] Y. B. Zel'dovich, Pis'ma Zh. Eksp. Teor. Fiz. 14, 270 (1971) [JETP Lett. 14, 180 (1971)].

[3] Y. B. Zel'dovich, Zh. Eksp. Teor. Fiz. 62, 2076 (1972) [Sov. Phys. JETP 35, 1085 (1972)].

[4] W. H. Press and S. A. Teukolsky, Nature (London) 238, 211 (1972).

[5] S. A. Teukolsky and W. H. Press, Astrophys. J. 193, 443 (1974).

[6] Ya. B. Zeldovich, J. Einasto, and S. F. Shandarin, Nature (London) 300, 407 (1982).

[7] R. Brito, V. Cardoso, and P. Pani, Superradiance: New Frontiers in Black Hole Physics, Vol. 971 (Springer, New York, 2020).

[8] T. Damour, N. Deruelle, and R. Ruffini, Lett. Nuovo Cimento 15, 257 (1976).

[9] S. Detweiler, Proc. R. Soc. Ser. A 352, 381 (1977).

[10] V. Cardoso, O. J. Dias, J. P. Lemos, and S. Yoshida, Phys. Rev. D 70, 044039 (2004); 70, 049903(E) (2004).

[11] R. Brito, V. Cardoso, and P. Pani, Classical Quantum Gravity 32, 134001 (2015).

[12] H. Witek, L. Gualtieri, P. Pani, and T. P. Sotiriou, Phys. Rev. D 99, 064035 (2019).

[13] W. E. East and F. Pretorius, Phys. Rev. Lett. 119, 041101 (2017).

[14] A. Arvanitaki, S. Dimopoulos, S. Dubovsky, N. Kaloper, and J. March-Russell, Phys. Rev. D 81, 123530 (2010).

[15] A. Arvanitaki and S. Dubovsky, Phys. Rev. D 83, 044026 (2011).

[16] C. Palomba et al., Phys. Rev. Lett. 123, 171101 (2019).

[17] S. J. Zhu, M. Baryakhtar, M. A. Papa, D. Tsuna, N. Kawanaka, and H.-B. Eggenstein, Phys. Rev. D 102, 063020 (2020).

[18] R. Brito, S. Ghosh, E. Barausse, E. Berti, V. Cardoso, I. Dvorkin, A. Klein, and P. Pani, Phys. Rev. Lett. 119, 131101 (2017).

[19] R. Brito, S. Ghosh, E. Barausse, E. Berti, V. Cardoso, I. Dvorkin, A. Klein, and P. Pani, Phys. Rev. D 96, 064050 (2017).

[20] L. Tsukada, T. Callister, A. Matas, and P. Meyers, Phys. Rev. D 99, 103015 (2019).

[21] L. Tsukada, R. Brito, W. E. East, and N. Siemonsen, Phys. Rev. D 103, 083005 (2021).

[22] R. Brito, S. Grillo, and P. Pani, Phys. Rev. Lett. 124, 211101 (2020).

[23] D. Ghosh and D. Sachdeva, Phys. Rev. D 103, 095028 (2021).

[24] M. C. Ferreira, C. F. B. Macedo, and V. Cardoso, Phys. Rev. D 96, 083017 (2017).
[25] O. A. Hannuksela, K. W. K. Wong, R. Brito, E. Berti, and T. G. F. Li, Nat. Astron., arXiv:1804.09659.

[26] J. Zhang and H. Yang, Phys. Rev. D 101, 043020 (2020).

[27] D. Baumann, H. S. Chia, R. A. Porto, and J. Stout, Phys. Rev. D 101, 083019 (2020).

[28] H. Fukuda and K. Nakayama, J. High Energy Phys. 01 (2020) 128.

[29] T. Ikeda, R. Brito, and V. Cardoso, Phys. Rev. Lett. 122, 081101 (2019).

[30] V. Cardoso, W.-d. Guo, C. F. Macedo, and P. Pani, Mon. Not. R. Astron. Soc. 503, 563 (2021).

[31] D. Blas and S. J. Witte, Phys. Rev. D 102, 123018 (2020).

[32] D. Blas and S. J. Witte, Phys. Rev. D 102, 103018 (2020).

[33] M. Baryakhtar, M. Galanis, R. Lasenby, and O. Simon, Phys. Rev. D 103, 095019 (2021).

[34] B. Holdom, Phys. Lett. 166B, 196 (1986).

[35] J. Jaeckel and A. Ringwald, Annu. Rev. Nucl. Part. Sci. 60, 405 (2010).

[36] R. Essig et al., in Proceedings, 2013 Community Summer Study on the Future of U.S. Particle Physics: Snowmass on the Mississippi (CSS2013): Minneapolis, MN, USA (2013) [arXiv:1311.0029].

[37] M. Fabbrichesi, E. Gabrielli, and G. Lanfranchi, arXiv: 2005.01515.

[38] B. Batell, M. Pospelov, and A. Ritz, Phys. Rev. D 80, 095024 (2009).

[39] J. M. Cline, G. Dupuis, Z. Liu, and W. Xue, J. High Energy Phys. 08 (2014) 131.

[40] P. Ko, W.-I. Park, and Y. Tang, J. Cosmol. Astropart. Phys. 09 (2014) 013.

[41] M. Escudero, S. J. Witte, and D. Hooper, J. Cosmol. Astropart. Phys. 11 (2017) 042.

[42] M. Pospelov, J. Pradler, J. T. Ruderman, and A. Urbano, Phys. Rev. Lett. 121, 031103 (2018).

[43] G. Mohlabeng, Phys. Rev. D 99, 115001 (2019).

[44] D. Hooper, R. K. Leane, Y.-D. Tsai, S. Wegsman, and S. J. Witte, J. High Energy Phys. 07 (2020) 163.

[45] A. Caputo, H. Liu, S. Mishra-Sharma, M. Pospelov, J. T. Ruderman, and A. Urbano, Phys. Rev. Lett. 127, 011102 (2021).

[46] G. Alonso-Álvarez, F. Ertas, J. Jaeckel, F. Kahlhoefer, and L. J. Thormaehlen, J. Cosmol. Astropart. Phys. 11 (2020) 029.

[47] I. M. Bloch, A. Caputo, R. Essig, D. Redigolo, M. Sholapurkar, and T. Volansky, J. High Energy Phys. 01 (2021) 178.

[48] A. Mirizzi, J. Redondo, and G. Sigl, J. Cosmol. Astropart. Phys. 03 (2009) 026. 
[49] S. Dubovsky and G. Hernández-Chifflet, J. Cosmol. Astropart. Phys. 12 (2015) 054.

[50] P. Pani, Int. J. Mod. Phys. A 28, 1340018 (2013).

[51] P. Pani, V. Cardoso, L. Gualtieri, E. Berti, and A. Ishibashi, Phys. Rev. Lett. 109, 131102 (2012).

[52] P. Pani, V. Cardoso, L. Gualtieri, E. Berti, and A. Ishibashi, Phys. Rev. D 86, 104017 (2012).

[53] M. Baryakhtar, R. Lasenby, and M. Teo, Phys. Rev. D 96, 035019 (2017).

[54] D. Baumann, H. S. Chia, J. Stout, and L. ter Haar, J. Cosmol. Astropart. Phys. 12 (2019) 006.

[55] V. Cardoso, O. J. Dias, G. S. Hartnett, M. Middleton, P. Pani, and J. E. Santos, J. Cosmol. Astropart. Phys. 03 (2018) 043.

[56] V. P. Frolov, P. Krtous, D. Kubiznak, and J. E. Santos, Phys. Rev. Lett. 120, 231103 (2018).

[57] S. R. Dolan, Phys. Rev. D 98, 104006 (2018).

[58] H. An, M. Pospelov, and J. Pradler, Phys. Rev. Lett. 111, 041302 (2013).

[59] E. Cannizzaro, A. Caputo, L. Sberna, and P. Pani, Phys. Rev. D 103, 124018 (2021).

[60] P. Pani and A. Loeb, Phys. Rev. D 88, 041301 (2013).

[61] J. P. Conlon and C. A. Herdeiro, Phys. Lett. B 780, 169 (2018).

[62] P. Kaw and J. Dawson, Phys. Fluids 13, 472 (1970).

[63] A. I. Akhiezer and R. V. Polovin, Soviet Phys. JETP 3 (1956), https://www.osti.gov/biblio/4361348.

[64] C. Max and F. Perkins, Phys. Rev. Lett. 27, 1342 (1971).

[65] G. Ficarra, P. Pani, and H. Witek, Phys. Rev. D 99, 104019 (2019).

[66] A. Caputo, H. Liu, S. Mishra-Sharma, and J. T. Ruderman, Phys. Rev. D 102, 103533 (2020).

[67] A. Caputo, H. Liu, S. Mishra-Sharma, and J. T. Ruderman, Phys. Rev. Lett. 125, 221303 (2020).

[68] S. D. McDermott and S. J. Witte, Phys. Rev. D 101, 063030 (2020).
[69] S. J. Witte, S. Rosauro-Alcaraz, S. D. McDermott, and V. Poulin, J. High Energy Phys. 06 (2020) 132.

[70] V. Cardoso, T. Ikeda, C. J. Moore, and C.-M. Yoo, Phys. Rev. D 97, 084013 (2018).

[71] A. Franceschini, G. Rodighiero, and M. Vaccari, Astron. Astrophys. 487, 837 (2008).

[72] W. Vestrand, Astrophys. J. 271, 304 (1983).

[73] M. S. Longair, High Energy Astrophysics (Cambridge University Press, 2011).

[74] A. Neronov and I. Vovk, Science 328, 73 (2010).

[75] E. Stueckelberg, Helv. Phys. Acta 11, 225 (1938).

[76] M. Reece, J. High Energy Phys. 07 (2019) 181.

[77] M. Ahlers, J. Jaeckel, J. Redondo, and A. Ringwald, Phys. Rev. D 78, 075005 (2008).

[78] J. S. Schwinger, Phys. Rev. 82, 664 (1951).

[79] M. Pospelov, Phys. Rev. D 80, 095002 (2009).

[80] M. Pospelov, A. Ritz, and M. B. Voloshin, Phys. Lett. B 662, 53 (2008).

[81] M. Dutra, M. Lindner, S. Profumo, F. S. Queiroz, W. Rodejohann, and C. Siqueira, J. Cosmol. Astropart. Phys. 03 (2018) 037.

[82] J. Schwinger, Phys. Rev. 82, 664 (1951).

[83] E. Adelberger, G. Dvali, and A. Gruzinov, Phys. Rev. Lett. 98, 010402 (2007).

[84] V. A. Kostelecký and S. Samuel, Phys. Rev. D 39, 683 (1989).

[85] L. Bonetti, L. R. dos Santos Filho, J. A. Helayël-Neto, and A. D. Spallicci, Phys. Lett. B 764, 203 (2017).

[86] E. R. Williams, J. E. Faller, and H. A. Hill, Phys. Rev. Lett. 26, 721 (1971).

[87] D. Ryutov, Plasma Phys. Controlled Fusion 39, A73 (1997).

[88] A. Retin, A. D. Spallicci, and A. Vaivads, Astropart. Phys. 82, 4955 (2016).

[89] L. Bonetti, J. Ellis, N. E. Mavromatos, A. S. Sakharov, E. K. Sarkisyan-Grinbaum, and A. D. A. M. Spallicci, Phys. Lett. B 768, 326 (2017). 